\title{
XIX. Defense and security
}

Defending and maintaining the security of a country and its population are critically important endeavors. These activities, pursued by both the military and civilian authorities of a country encompass a broad range of activities including anticipation, detection and response to threats to the safety of a country and its citizens. As in other domains, nanotechnology can make valuable contributions, either by improving existing technologies or by introducing new ones. Apart from its direct contributions to defense and security activities, the development of nanotechnology contributes to maintaining a country's technological parity with respect to other countries.

\section{Defense}

For the past several decades, sophisticated technological developments in the areas of defense and security have become increasingly reliant on dual-use technologies that can be used in both military and civilian applications. This evolution reflects the fact that research on and development of, these technologies are extremely expensive. The vast and diverse capabilities in the civilian domain insure rather rapid progress and economy of scale for civilian associated technologies. Research and maintenance costs can be reduced by using commercially developed components in defense and security applications, where possible. Information et communication technologies already rely heavily on nanotechnology since the integrated circuits are designed with an accuracy that is now in the range 10-30 $\mathrm{nm}$

Defense activities benefit strongly from applications of four main areas of nanotechnology. (figure 137).

A few results of applying nanotechnology to defense needs are:

- Helmet sensors and wireless networks providing information and communication applications for soldiers.

- Hybrid power and nanocatalysts for energy applications in military vehicles

- Self-healing structures and biomimic lightweight materials for aeronautics and targeted drug delivery techniques for soldiers derived from life science research. 


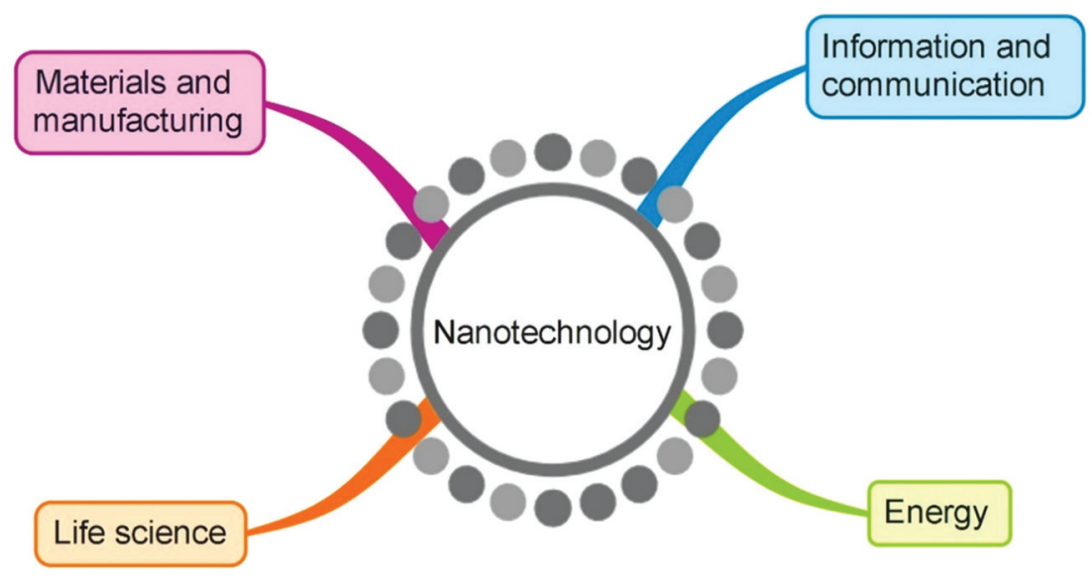

Figure 137. Four main areas of nanotechnology utilized for defense.

- High strength nanomaterials for satellites and adaptive structures for planes or missiles.

- Microelectronics systems which are now designed at the nanoscale and present in all information and communication systems.

- Lower weight portable weapons

- Guns that detect, target and fire automatically with self-guided bullets.

\section{The modern warrior}

Military equipment is evolving rapidly. The equipment of today's warrior is very different from that used during World War II or the Vietnam war. That of future warriors will be even more advanced. These warriors will be connected in real time with other soldiers and with their logistical support units. This will give a group of soldiers a shared intelligence making their action more efficient. Furthermore, this will allow continuous monitoring of the health and mental state of each soldier.

Figure 138 shows areas where nanotechnology can contribute to the equipment of the future soldier.

The evolution of battle gear is towards a lightweight, comfortable battle suit with temperature and moisture control that embeds high-technology capabilities and provides better protection against various projectiles. By high technology we mean sensors and electronic devices that allow communication, that monitor health and that can react immediately to chemical or biological agents. The battle suit can be made ultrahydrophobic by depositing nanolayers of teflon. 


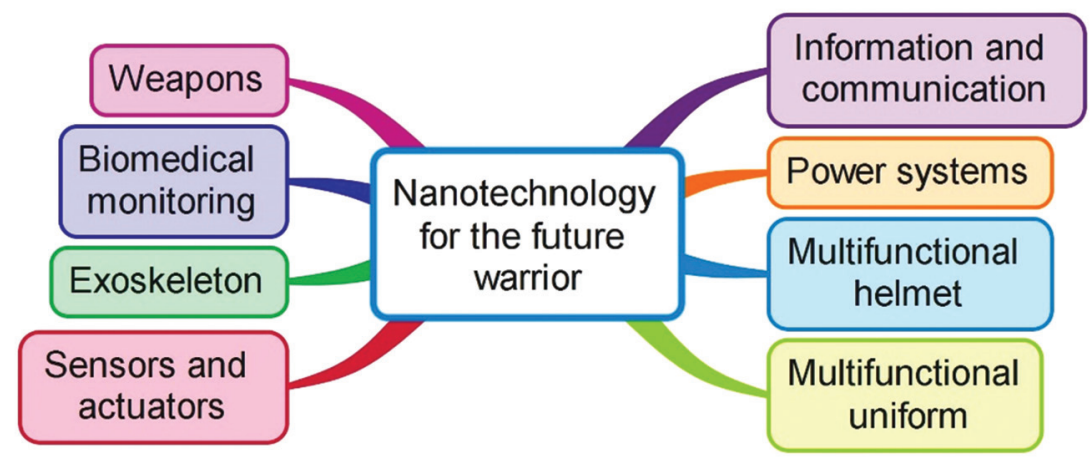

Figure 138. Nanotechnology is involved in providing many components of the equipment of the future soldier.

Protection against bullet damage or stabbing can be provided by "liquid body armor" consisting of a Kevlar vest containing a shear thickening fluid made of nanoparticles suspended in a liquid. Under normal conditions the fluid is easily deformable. But, upon impact, the fluid stiffens and undergoes a transition to a rigid material that prevents the projectile from penetrating into the body of the soldier. The force of impact of the bullet is distributed over a large area reducing the injury due to the kinetic energy of the projectile.

Nanofabrics can be designed for active camouflage in analogy to that existing in nature such as that of the cuttlefish for example. Active camouflage is based on panels and coatings that change appearance depending on the surroundings. The ultimate goal is to create an invisibility cloak. Research in this direction is based on negative-index metamaterials that we have already discussed.

As far as life sciences is concerned, nanotechnology will facilitate heath treatment and body repair. Smart biocompatible implants will sense the health status of the soldier and actuate targeted drug delivery by functionalized nanoparticles in case of health problems. Techniques for regenerative medicine for wound healing and, to some extent, to repair or rebuild organs and other parts of the body are also under development.

Since the equipment of the soldier will become more and more sophisticated and the soldier will also carry sophisticated weapons, minimization of weight is also an important issue. In addition, to power high tech portable equipment requires appropriate energy sources. Several solutions using nanotechnology continue to be investigated: lightweight energy sources such as batteries, flexible solar cells, devices to harvest vibrational energy, or thermal energy for low power applications. Micro fuel cells and micro nuclear batteries are also among the possibilities. 


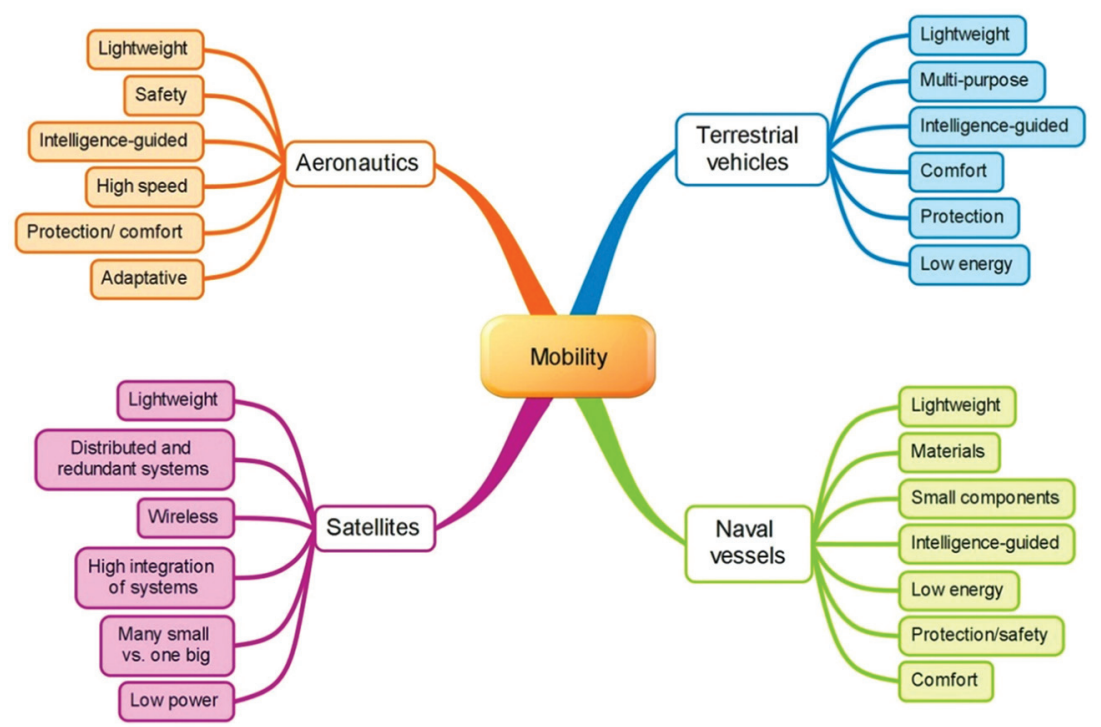

Figure 139. Aspects of military mobility to which nanotechnology can contribute. Created using the data of Simonis and Schilthuizen, Nanotechnology, innovation opportunities for tomorrow's defense, TNO Science and Industry, 2006.

\section{Mobility}

Mobility is an essential element of military tactics. This includes the use of terrestrial vehicles, naval vessels, aeronautics and satellites. Figure 139 indicates the areas in which nanotechnology can be employed to improve military mobility.

Lightweight vehicles can be constructed using nanocomposite plastics to replace metal. Another advantage derived from this is reduction of radar signature.

Providing adequate energy to the vehicle is always an issue. Energy requirements can be reduced by development of low energy consumption vehicles and low power consumption electronic equipment. As discussed in chapter 17, nanotechnology is already contributing to design of energy sources such as batteries, fuel cells, etc.. Nanocoatings are used to reduce wear in engines. The aerodynamic drag of fast vehicles such as planes or missiles can be reduced using nanocoatings and nanocomposites.

In aeronautics, there is a demand for vehicles with small radar and thermal signatures. Nanotechnology can provide radar absorptive coatings and high energy propellants based on nanodispersed aluminum. 


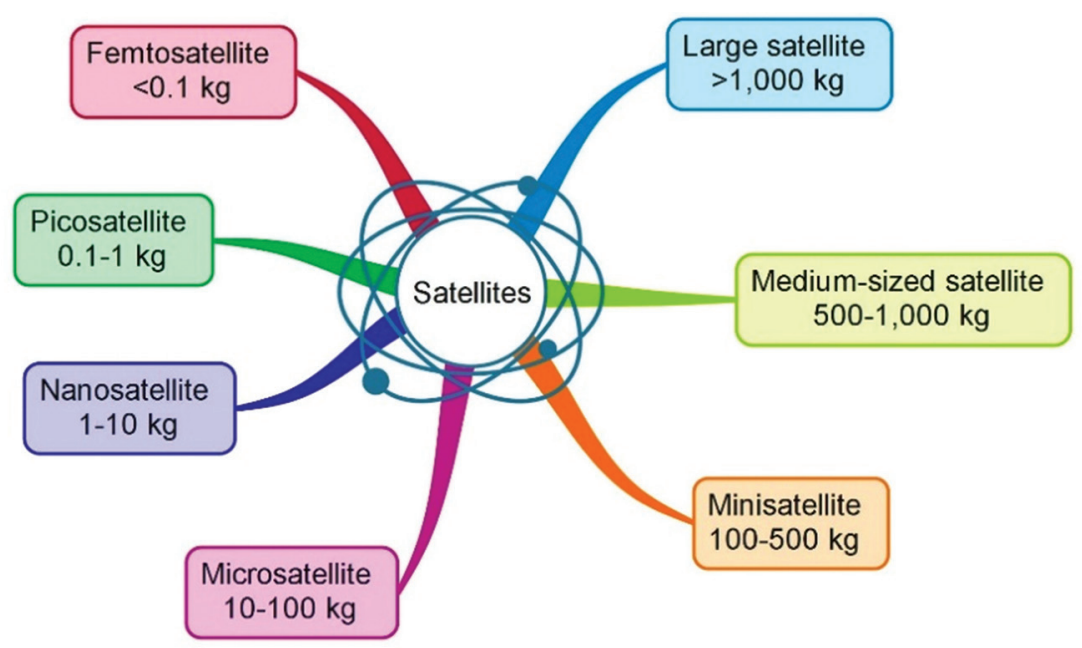

Figure 140. Classification of satellites according to their deployed weights (data from www.daviddarling.info).

Satellites are extensively used for observation and telecommunication. The use of nanocomposites can greatly reduce weight, a critical issue for the equipment carried by satellites. There is an increasing trend to rely on orbiting networks of small satellites rather than a single large satellite. A classification of satellites according to their deployed weight is shown in figure 140.

The nanotechnology based technique of quantum cryptography has a great potential for secure communications. It is based on quantum physics and the entanglement effect. With this technique one knows for sure that no one has read or modified the information before it is received.

\section{Weapons}

Modern weapons can be classified into two main categories: nonlethal and lethal. The goal of nonlethal weapons is to temporarily neutralize an enemy. They are mostly used by the police and security services. Lethal weapons are those capable of producing great bodily harm or death. The goals of weapon development are to make them even more effective while increasing precision, targeting and intelligence.

Nanotechnology enters in several areas of weapon development as is illustrated in figure 141. 


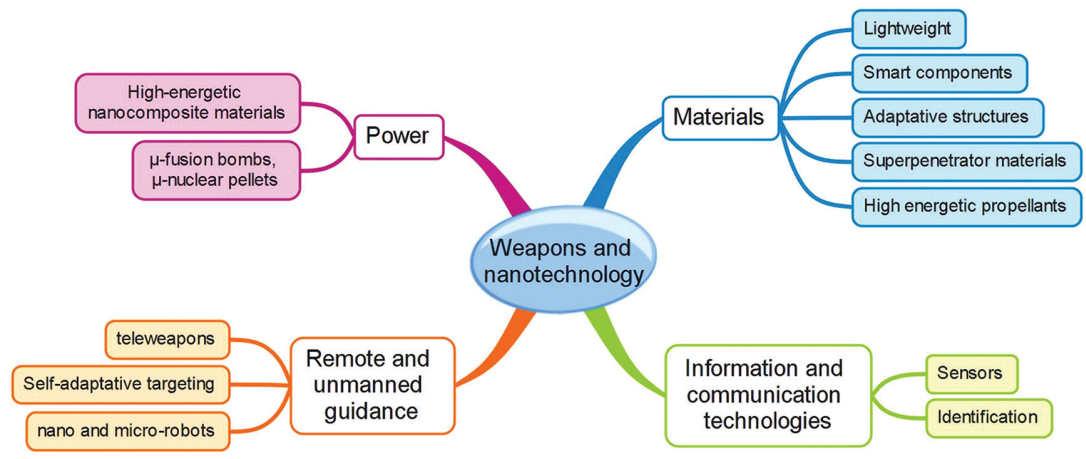

Figure 141. Areas where nanotechnology can improve weapons.

\section{Homeland security}

We live in a dangerous world. Natural disasters such as fires, floods, cyclones, typhoons, tornados or earthquakes can lead to great damage and disorganize societies. Terrorist attacks, executed by fanatics, are difficult to defend against (who, where, how and when?). They create a great sense of insecurity in the population. Anticipating and dealing with these threats and dangers is difficult. Technology can help. Within the technology sector, nanotechnology plays an increasing role.

Mitigating threats requires timely actions. To protect people and equipment threats must be detected, appropriate responses must be made and in the event that damages occur repairs must be made. The different aspects of threat mitigation are illustrated in figure 142.

\section{Detection}

Detection of the threats is essential to prevention. Appropriate sensors and analytical devices can provide early warnings of possible threats and allow intervention in a timely manner. In the event of a successful attack, similar analytical techniques may be employed in forensics investigations of the incident. This can be important in preventing recurrences and in pursuing perpetrators of these incidents as well as the detection of the nature of the products that have been used when it is the case. The main sectors in which analytical detection techniques are applicable are indicated in figure 143.

Releases of toxic chemicals can present serious threats to a community. This is true whether they are accidental or deliberate releases. 


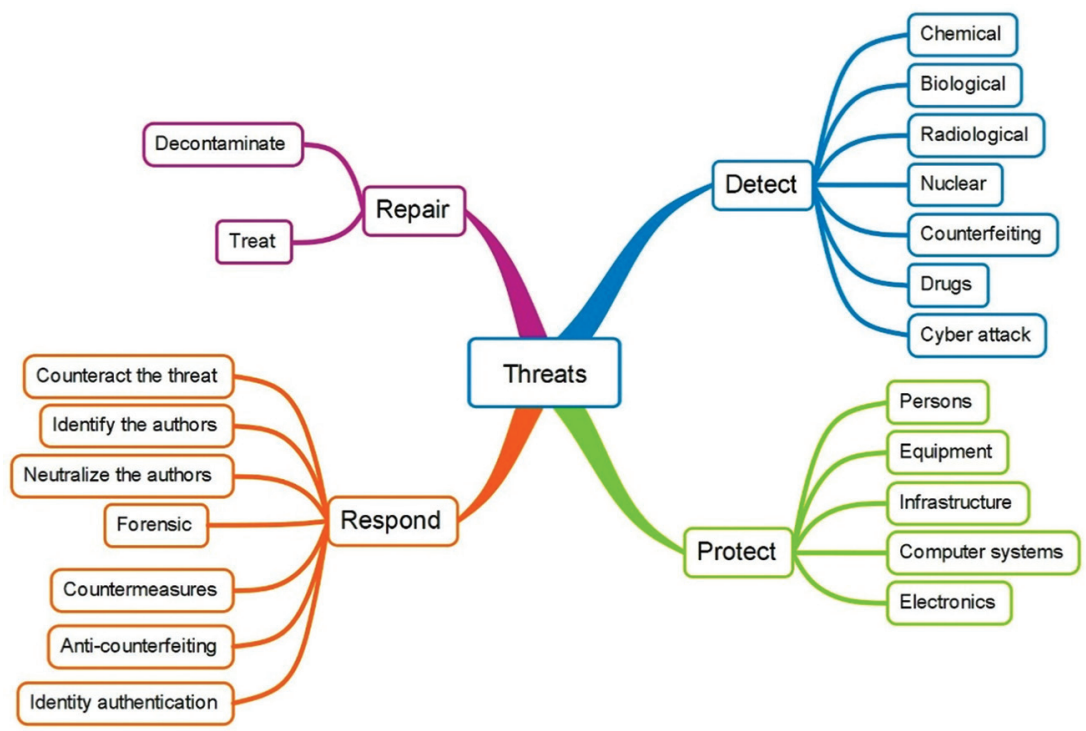

Figure 142. Threat mitigation.

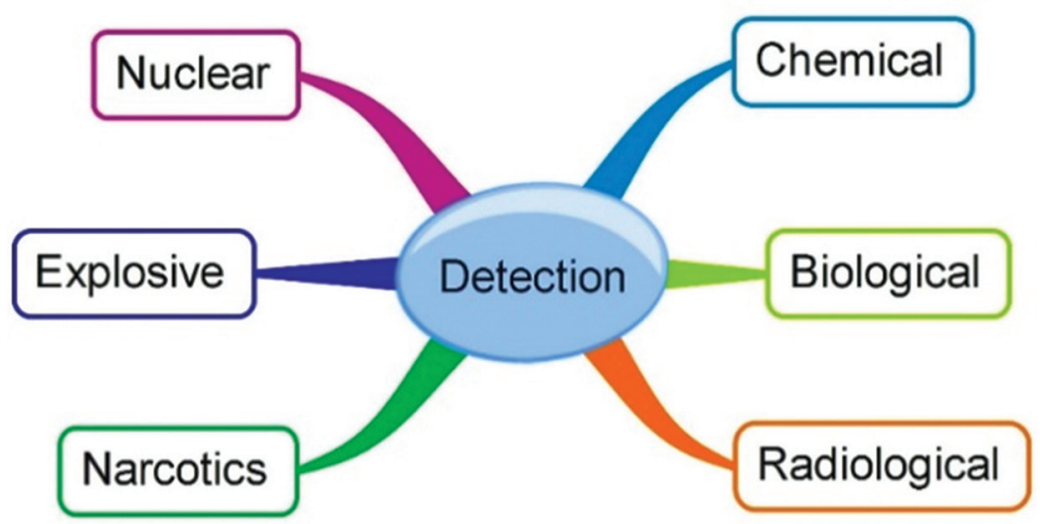

Figure 143. Sectors where detection is required.

The Observatory nanoproject has developed the classification of dangerous toxins presented in Figure 144.

A nerve agent can be sarin, tabun, cyclosarin, etc. Phosgene and chlorine are examples of choking agents. Blister agents include mustard gas or phosgene oxime, commonly known as CX. Cytoxis agents such as ricin or abrin are extremely toxic. In the case of accidents in the chemical industry, a number of harmful agents such as hydrogen cyanide, mercury or phosgene 


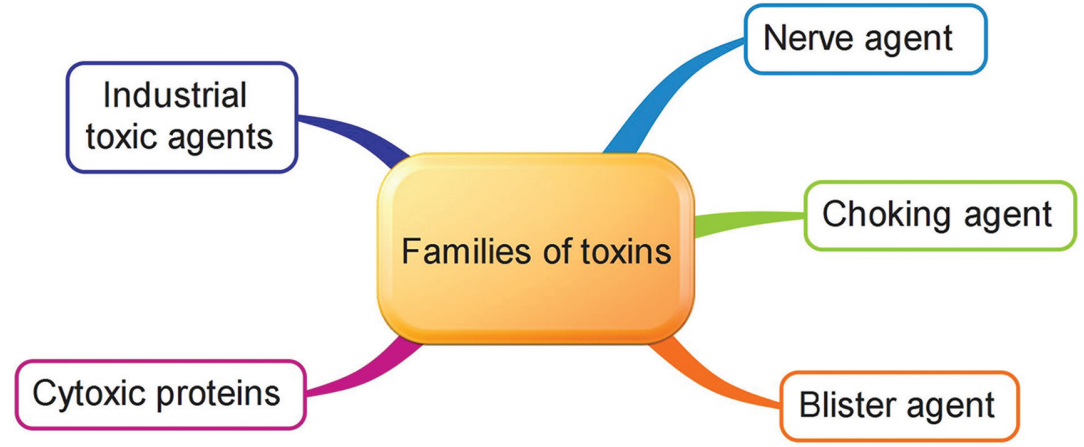

Figure 144. Different families of toxins. Classification from the ObervatoryNano project.

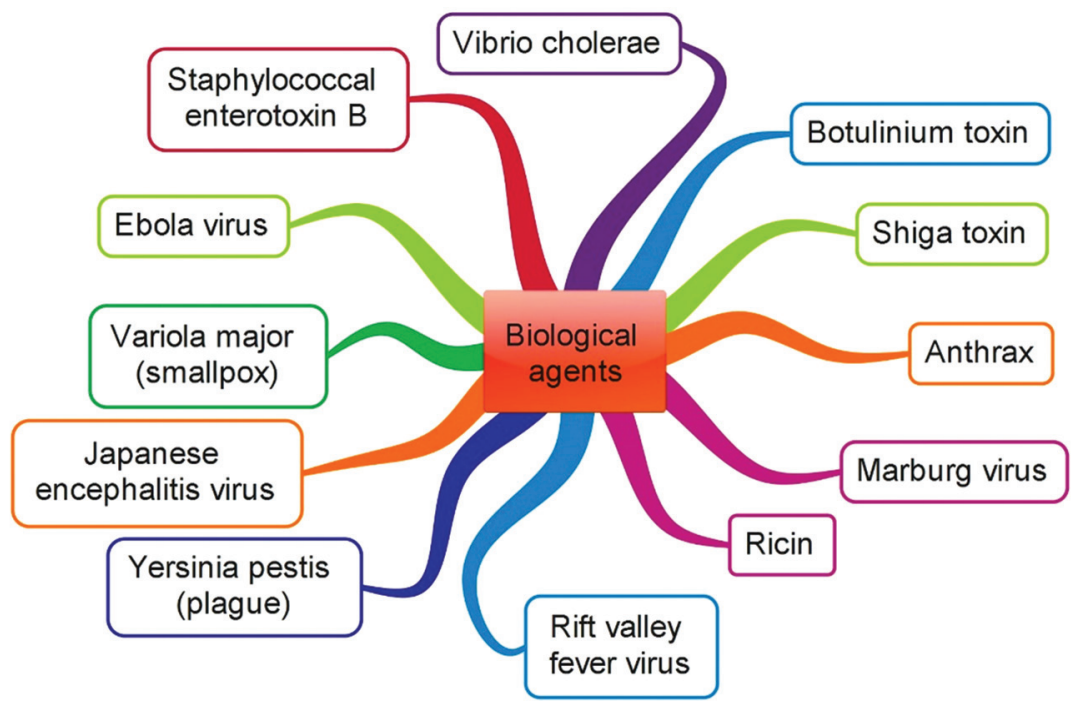

Figure 145. Examples of biological agents that could be used in biological weapons.

might be emitted. High sensitivity, material specific, and cheap sensors are required for these different toxins.

Viruses, bacteria and toxins are biological threats that can be spread among the human population, livestock, crops, food or water supplies in a terrorist attack. Some of the biological agents which can be potentially used as biological weapons are shown in figure 145. Recognition of these agents is based primarily upon nucleic acid and immunological detection techniques but physical and chemical techniques can also be useful. 
Nanotechnology and nanomaterials including nanoparticles, carbon nanotubes, and quantum dots are already being employed in several currently available sensors developed for toxins and biological agents. The small sizes of nanoparticles, nanotubes and nanowires means that the electrical properties of the sensing elements are sensitive to only a few molecules.

Nuclear proliferation and the possibility of misappropriation of a nuclear weapon or dispersal of radioactive matter using radioactive materials and conventional explosives constitute major threats in the modern world. Very sensitive detectors for nuclear materials exist but there is a need to have cheap and portable nuclear detectors. High resolution germanium detectors operating at low temperature are excellent gamma ray detectors but are expensive. Nanowire arrays of detectors are useful for imaging techniques and high counting rates. Alternative low cost detectors based on nanomaterials operating at room temperature can be useful to make quick measurements even if the resolution is not as good.

Detecting explosive materials in cargo, vehicles, luggage, aircraft etc. is a high priority objective of security forces. More than one hundred different types of explosives can be used and it is necessary that they be detected quickly and efficiently. Several detection techniques are employed in this sector. A number of them rely on the fact that absorption of volatile molecules found in the explosive modifies a physical or chemical property of the sensor. Nanocatalysis is often part of the detection process.

Narcotics detection requires cheap, selective, sensitive, fast and small sensors that can be operated under various conditions of temperature and humidity. The techniques utilized are similar to those generally used in chemical detection. The detection of narcotics is also a sector where nanotechnology and nanomaterials are involved. For example, selective membranes of nanomaterials are used to detect fentanyl that is forty time more potent than heroin.

\section{Protection}

Protecting people, infrastructure and equipment during and after explosions or chemical, biological and radiological accidents or attacks requires a variety of measures to be taken.

Some protection can be offered by proper clothing. Protective clothes should be affordable, lightweight and durable. Nanocomposites are 
expected to play an increasing role in this area. Nanofibers have large active areas that can be functionalized in order to provide a better protection against aerosols. Fabrics made out of carbon nanotubes offer a better shielding against explosives and bullets and are lighter than fabrics based on steel. Magnesium oxide nanoparticles loaded into nanofibers turn out to be better protective adsorbents than activated charcoal used today.

Buildings can be destroyed or damaged by explosions, fire, earthquakes, tsunamis, etc. Electronic infrastructure including communications, data processing and control equipment can also be partially or totally destroyed in an electromagnetic attack (E-bomb). Incorporation of nanomaterials in concrete or steel alloys increases the strength of these materials against ballistic projectiles. Carbon nanotubes or inorganic fullerenes can reinforce structures against explosions, or earthquakes. Nanocoatings or nanoadditives can improve the fire resistance of materials. Electromagnetic shielding with conducting polymers or nanocomposites containing conductive polymers can protect electronic equipment against electromagnetic attacks.

\section{Response}

Identification of dangerous individuals and the ability to detect them when they travel around the world is a first response to potential threats. There are several more or less complex methods for authentication of the identities of individuals: fingerprints, DNA, iris, retinal or facial identification, etc. Nanocomposites can be used as a medium to record biometric information.

Response to a security breech includes counter measures such as identification and neutralization of those responsible, minimization of negative effects and taking appropriate actions to prevent reoccurrence. Forensic analysis of evidence at crime scenes has become more and more efficient thanks to new techniques of investigation. Forensics aims to determine the origin and causal links for a crime or a terrorist attack. A number of the techniques employed rely on nanotechnology that enhances the analysis capabilities and give new possibilities to pin down relevant indices that can be used to expose the persons responsible.

As an illustration of a device issued from nanoscience, which has major applications in forensics, figure 146 shows applications of the scanning 


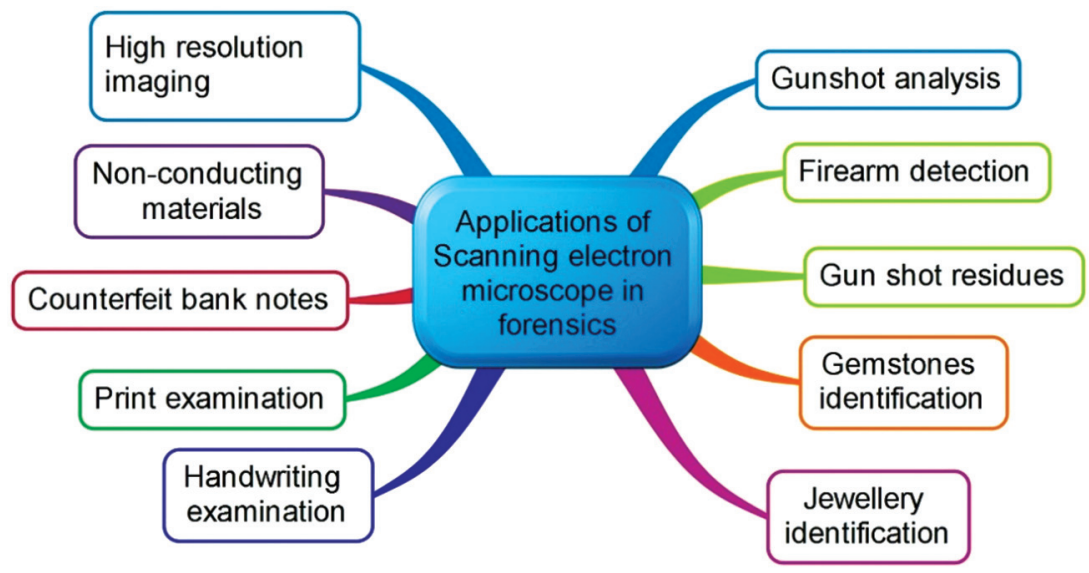

Figure 146. Some applications of scanning electron microscopy in forensics. Examples from OservatoryNano project.

electron microscope. Depending upon the technique used in the microscope, spatial resolutions in the nanometer range can be achieved.

A study by the OECD has estimated that, in 2007, international trade of counterfeit and pirated goods amounted to about 250 billion dollars, representing around $2 \%$ of global trade in that year. Many of the solutions used to protect products and consumers against counterfeiting are based on nanotechnology. Cheap intelligent materials and devices, DNA tags for example, can be used to label products and their packaging. There is an increasing demand to trace products all the way from the manufacturer to the consumer. Positioning and localization techniques based on electronic devices are used to do that. Radio frequency identification tags (RFID) are now widely used to identify objects. Metal nanoparticles or carbon nanotubes improve their operating characteristics.

The main anti-counterfeiting technologies based on nanotechnology are shown in figure 147 together with their areas of application.

\section{Repair}

After a terrorist attack or a natural disaster, repairs must be made. People injured during a chemical, biological, radiological dispersive devices, nuclear and explosive (CBRNE) attacks need medical aid and decontamination. Decontamination is also necessary in the case where the environment has been compromised. 


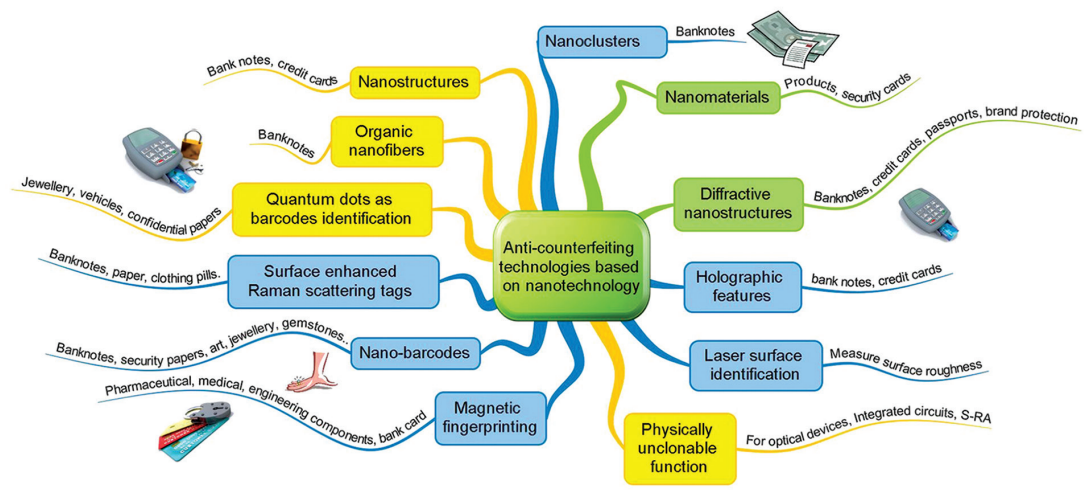

Figure 147. Main anticounterfeiting techniques based on nanotechnology (from the ObservatoryNano project). Techniques which are commercial or pre-commercial are in blue. Techniques at the prototype level are in green. Techniques in the research stage are in yellow.

Nanoparticles and nanomaterials are effective decontamination agents because of their high reactivities and large surface areas. They can be used to decontaminate chemical warfare agents. Nanosized metal oxides of aluminum, titanium and cerium can be used for decontamination and disinfection. Nanocrystals of magnesium oxide can absorb organophosphorous compounds. Their advantage compared to activated charcoal is that they react much faster and destroy the dangerous compound rather than simply physisorb it. Nanoscale powders of magnesium and calcium oxides have antimicrobial properties. They can destroy vegetative cells of Escherichia coli and several bacilli. Other specific nanoparticles are also used to quickly decontaminate objects after bacillus anthracis attacks or Escherichia coli contaminations.

Titanium dioxide $\left(\mathrm{TiO}_{2}\right)$ can be used as a photocatalyst for pollutant destruction. A great advantage is that it is inexpensive and non-toxic. Using $\mathrm{TiO}_{2}$, it is possible to manufacture photocatalytic nanowires that can be transformed into free standing membranes which can be used in filters for gas masks and to block toxic gases. Many other possibilities exist for decontamination based on nanoparticles or nanomaterials. A deeper description of these possibilities can be found in the ObservatoryNano report. 


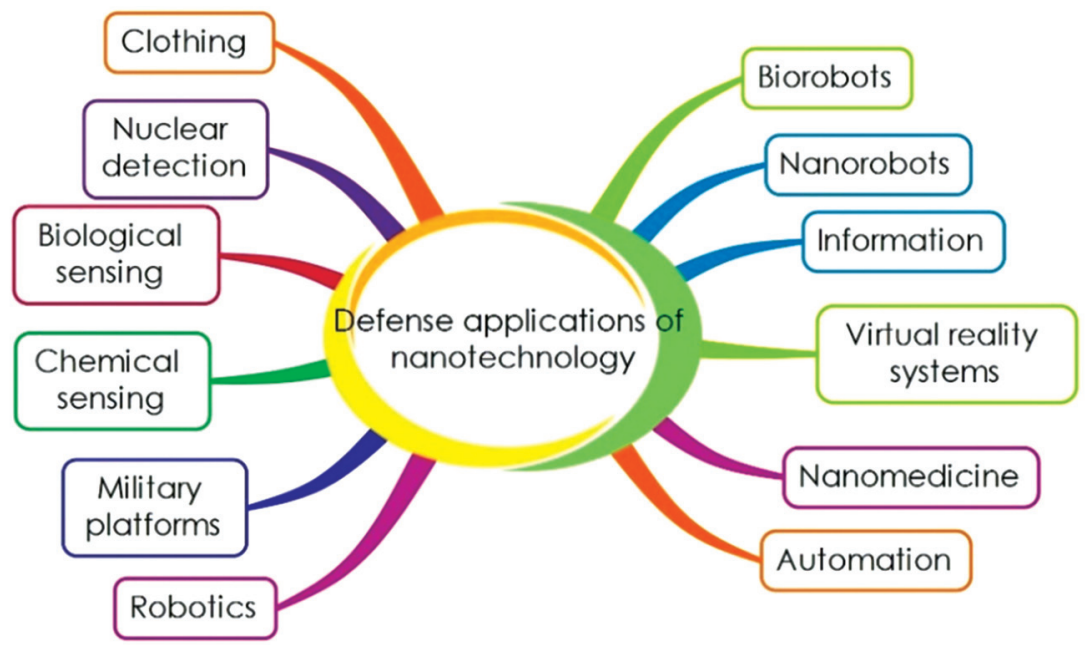

Figure 148. Main sectors in the defense and security domains where nanotechnology can play a role.

\section{Summary}

Nanotechnology is already playing a major role in defense and security applications. This will be amplified in the future and most future military equipment will employ some nanotechnology. An irreversible evolution towards dual-use technologies is observed reflecting cost/benefit analyses and rapid progress in civilian research and development efforts. The keywords in the defense and security domains are smaller, faster, lighter and cheaper. Nanotechnology helps us to address all of these imperatives. Some current and potential applications of nanotechnology to defense or security are summarized in figure 148 .

Homeland security is an overriding concern in modern societies. The primary goal is to anticipate and prevent or at least reduce the impact of terrorist attacks and natural disasters. It is a difficult goal. It relies on detection of threats and a correct treatment of the consequences of attacks or disasters. Technology is a critical tool in this respect. Nanotechnology offers new opportunities to develop cheap, small and smart, fast and selective, sensors. Nanoparticles and nanomaterials are of great help in detecting and decontaminating dangerous or deadly agents. 